\title{
Machine Learning Assisted EDFA Gain Ripple Modelling for Accurate QoT Estimation Ankush Mahajan ${ }^{*}$, Kostas Christodoulopoulos ${ }^{3}$, Ricardo Martinez ${ }^{1}$, Salvatore Spadaro², Raul Munoz ${ }^{1}$
}

\author{
${ }^{1}$ Catalan Telecommunications Technology Center (CTTC), Barcelona, Spain \\ ${ }^{2}$ Polytechnic University of Catalonia (UPC), Barcelona, Spain \\ ${ }^{3}$ Nokia Bell Labs, Stuttgart, Germany \\ *ankush.mahajan@cttc.cat
}

Keywords: EDFA, QoT, MACHINE LEARNING

\begin{abstract}
Wavelength dependent EDFA gain ripple has an impact on connection's OSNR performance. We propose a machine learning regression model to estimate the end to end gain ripple penalty and to increase QoT estimation accuracy.
\end{abstract}

\section{Introduction}

The emergence of Elastic Optical Networks (EONs) has introduced flexibility in the optical transport supporting heterogeneous data rates, optical spectrum channels, modulation formats, etc. This leads to attain higher spectral efficiency and capacity, while keeping the network costs as low as possible [1]. For reliable and efficient optical network planning and operation, accurate estimation of Quality of Transmission (QoT) before establishing the connections is necessary.

Traditionally, QoT estimation is performed with some analytical Physical Layer Model (PLM), while, recently Machine Learning (ML)-based estimation [2-4] has gained a lot of attention. The main sources of noise accounted in these QoT estimators are the Amplified Spontaneous Emission (ASE) noise generated at both span and node amplifiers and the Non Linear Interference (NLI) noise, which considers fiber non linearities, self and cross channel interferences. Typically, an additional design margin is adopted and applied to the QoT tool to accommodate estimation errors and other uncertainty parameters [5]. Removing such uncertainties would allow to reduce the margin from the QoT tool without compromising the QoT estimation accuracy. By doing this, appealing advantages such as higher efficiency and/or lower cost can be achieved during the network planning and upgrading phases. Erbium Doped Fiber Amplifiers (EDFAs) are key devices in WDM and EON transport networks to ensure the required connection QoT level at the receivers. Nevertheless, EDFAs are the dominant noise source (ASE) in those networks. Typically, span EDFAs are operated in Automatic Gain Controlled (AGC) mode with near to zero tilt (first order/ linear correction) to get a flat gain in the C-band as shown in Fig. 1(a) [6]. However, although the gain tilt profile is maintained at zero still there are gain fluctuations/ripples within the gain bandwidth of EDFAs [7]. These gain ripple effects may be due to: i) imperfections in the gain flattening filters at the amplifier output; or ii) wavelength dependent absorption/ emission coefficients of $\boldsymbol{E r}^{3+}$ ions [8].

In light of the above herein, we investigate the contribution of the wavelength dependent EDFA gain ripple on the QoT. We then propose a ML regression model based on a link formulation that leverages monitoring data of established connections to estimate the gain ripple penalty for new connection requests. Keeping realistic simulation environment, we observe a related margin reduction from $1.02 \mathrm{~dB}$ to $0.08 \mathrm{~dB}$ in optical signal to noise ratio (OSNR).

\section{Related Work}

Several analytical models were introduced to estimate EDFA wavelength dependent gain and channel power at EDFA output, under different channel loading conditions. The models range from limited accuracy and simple characterization to very detailed estimations, trading-off processing time for accuracy [9]. Still even the most detailed models require knowledge of certain physical parameters, that might be hard to measure or known in a deployed network. Some recent ML-based works addressed the wavelength dependent gain spectra estimation [10] or the power excursion during dynamic add/drop [11]. The dataset used for training those models were either i) analytical without considering fiber effects/NLI noise contribution, or ii) experimental, requiring the collection of data for each individual span EDFA, which practically limits the usability of these approaches. In [12] the gain offset of a AGC-EDFA was experimentally investigated and a method was derived aiming at reducing the gain offset up to 0.5 to $0.7 \mathrm{~dB}$ when encompassing up to five cascaded EDFA spans. The scenario targeted in [12] was dynamic with add/drop of new/existing connections. However, for fixed load EDFAs, with static network conditions, the gain ripples effect was not explored. Moreover, optical attenuators were used to replace fibers in experiments and hence the effects of NLI noise contribution were not considered at all. In [10], a neural network based EDFA gain model is proposed to model an individual EDFA gain profile. This approach is again limited to per span EDFA modelling (18000 channel loading conditions for single EDFA). EDFA cascading and its effect on gain ripples were also not considered.

In brief, to the best of our knowledge, the behaviour of EDFA gain ripple penalty and its effect on QoT estimation still need to be investigated. Therefore, we devise an estimation tool that can predict EDFA gain ripple penalty for new connection based on training from monitored data of established ones. 


\section{Methodology and Proposed Solution}

We consider a Flexi-grid EON with Reconfigurable Optical Add/Drop Multiplexers (ROADMs) connected through uncompensated fiber links. Each link consists of multiple fiber spans that terminate at an EDFA to compensate the span loss. We assumed that span EDFAs are operated in AGC mode with zero tilt having gain ripple profiles. We consider Dynamic Gain Equalizers that flatten the gain ripples at every ROADM node, so at the end of each link. To understand the trends of gain ripple profiles, we performed experiments on EDFAs to capture the gain fluctuations in the optical spectrum band of $\sim 1530$ - 1563nm (40 channels). All EDFAs used in experiments were operated in AGC mode with zero tilt by preadjusting their operating points. Based on collected experimental data, we created realistic sets of individual span EDFA gain profiles. We denote these gain profiles as $G_{i}(\lambda)$ having an average gain value of $G_{i, a v g}$ and wavelength dependent ripple, $G_{i, R}(\lambda)$ given by Eq. 1 .

$G_{i}(\lambda)=G_{i, a v g}+G_{i, R}(\lambda)$ where $i$ represent profile index

(1)

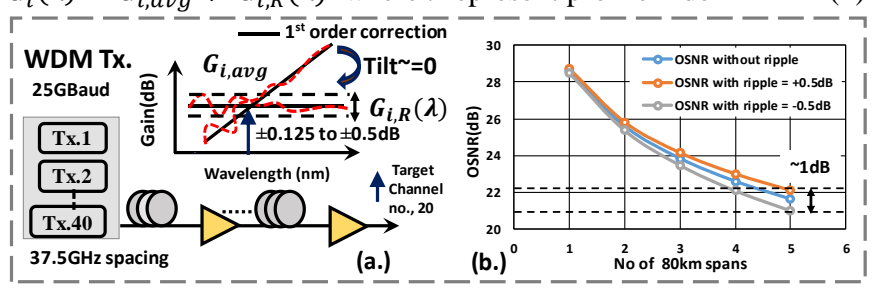

Fig 1. (a) Adopted setup, (b) OSNR fluctuation of $\sim 1 \mathrm{~dB}$ observed due to the EDFA gain ripple after 5 spans.

We assign these ripples profiles to span EDFAs and we emulated a static link set up in VPI Transmission Maker version 9.9 as shown in Fig. 1(a). We found $\sim 1 \mathrm{~dB}$ (see Fig.1(b)) of fluctuation with increasing number of spans. We then extended these static link simulation experiments in entire C-band to capture the effect of gain ripples on OSNR.

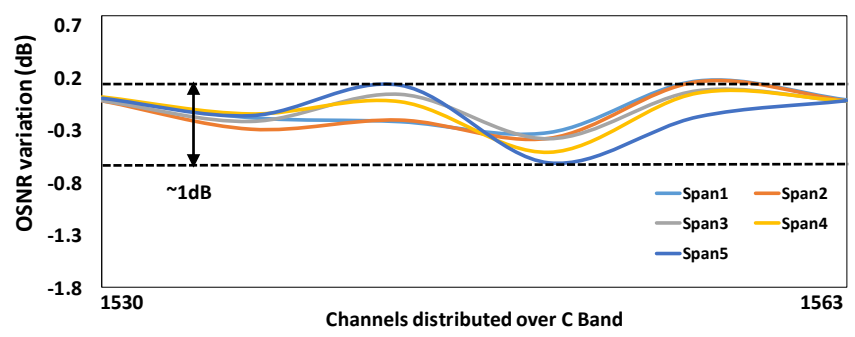

Fig 2. EDFA gain ripple effect on $\operatorname{OSNR}(\mathrm{dB})$ over the C-band.

Fig. 2 represents the relative OSNR variation distribution over 40 channels in C-band with respect to flat EDFA profiles. We observe that with the cascade of spans $(80 \mathrm{~km}$ length used in simulations), the OSNR variation increases ( $1 \mathrm{~dB}$ in C-band as also shown in Fig. 1(b)). The trend in Fig. 2 indicates less fluctuation on spectrum band edge channels. This is because the ripple profile used contains more rapid fluctuations in the center, keeping $G_{i, a v g}$ constant. The shape of the ripple can vary over longer time (aging), leading to higher peak to peak variations, but this is slowly time varying. So in short and medium term there is clear trend which makes modelling of these variations possible. These variations impact the accuracy of the QoT estimator within a margin range as described in Fig. 2 and Fig. 7 of [13].
If we use a physical layer model (PLM), such as the Gaussian Noise-GN model, the typical assumption is a flat EDFA gain [14] requiring a high design margins as given by Eq. 2

$\operatorname{OSNR}_{\text {Flat }}(\lambda)=\frac{P_{O}(\lambda)}{G_{\text {Noise_flat_p }}}+$ design margin $_{1}$

where $G_{\text {Noise flat } p}$ corresponds to the "total noise" of the path estimated by the PLM having no EDFA gain ripple information. We call it as, "ripple unaware PLM". The penalty due to gain ripple fluctuations is within the design margin $_{1}$ (which is flat and equal to the worst case). In this case, we had to model all EDFAs in the network in a calibration face, which would be time consuming and would need to be repeated, when the ripple function changes (aging). In this work, we use the well accepted GN model as PLM. We extend the ripple unaware PLM model to capture the ripple penalties to ultimately reduce the required margin for new connections (ripple aware PLM). We considered accumulated ripple penalties at link ends which are then added over the path. Our extended ripple aware PLM estimates OSNR by Eq. 3

$\operatorname{OSNR}_{\text {Ripple }}(\lambda)=\frac{P_{O}(\lambda)}{G_{\text {Noise_ripple_p }}(\lambda)}+$ design margin $_{2}$

where $G_{\text {Noise_ripple_p }}(\lambda)=G_{\text {Noise_flat_p }}+G_{\text {ripple_p }}(\lambda)$ in this case is not flat but models the wavelength $(\lambda)$ dependent ripple noise leading to lower margin (design meagin 2 < design meagin $_{1}$ ). To improve in this way, the accuracy of QoT estimation, we use monitoring information from Optical Performance Monitors (OPMs) assumed to be installed at the end of each link [15] and at the end of connections. Such information is used to fit per link noise ripple penalty functions, which in turn are used to calculate the end-to-end ripple penalties, $G_{\text {ripple_p }}(\lambda)$.

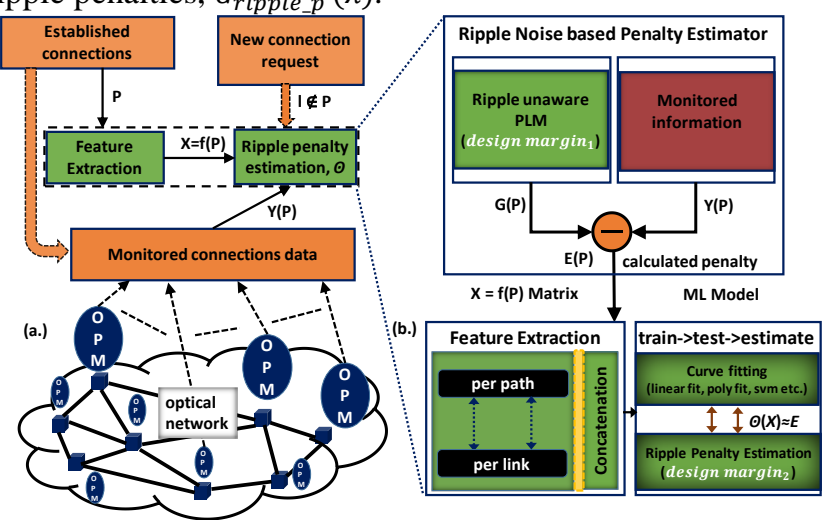

Fig 3. (a) Overall Flowchart and architecture, (b) ML-based penalty estimator (i.e., train/test/estimate).

We assume an optical network with established connections and their attributes (also referred as state of network at a given time) denoted by $P$. We assume a ripple unaware PLM model as represented in Eq. 2, which calculates the noise of the established connections end-to-end as $G_{\text {noise_flat_p }}(P)$, and the related per link noise as $G_{\text {noise flat } l}(P)$. Note that $P$ contains attributes for a connection such as, the traversed links, central wavelength etc. We also assume that we monitor the OSNR of the established connections and thus their noise at the path level $Y_{p}(P)$ and at the link level $Y_{l}(P)$ and store it in QoT tool database. This data serves as the ground truth, it defines the true $G_{\text {Noise_ripple_p }}(\lambda)$, so with zero margin. The flowchart representing the integration between collected monitoring information and the ML assisted penalty estimation function is depicted in Fig. 3(a). 
We denote the difference of $Y$ and $G_{\text {noise }}$ flat as i) $E_{l}(P)=Y_{l}(P)$ $G_{\text {noise_flat_l }}(P)$ which is a vector with the ripple penalties at the end of each link, accumulated over the links, spans and ii) $E_{p}(P)=Y_{p}(P)-G_{\text {noise_flat_p }}(P)$ which is a vector with the ripple penalties at connections' end, accumulated over all used links. We let $E$ be the concatenation of both penalty vectors $E_{l}$ and $E_{p}$. From corresponding connections attributes, $P$, we extract per link and per path features matrices. Additionally, a bias term (per link) is also considered to account the monitoring error and for non-zero equalized ripple. The per link and per path features are merged into a single features matrix, $X=f(P)$. Our goal is to identify the function $G_{\text {ripple_p }}=\mathcal{O}(X) \approx E$ that maps well the features matrix $X$ to the penalty $E$ generated due to the gain ripple. We rely on ML for training and fitting of $X$ on $E$ and finding the function $\odot$. Assuming a new connection request $l \notin P$, we will use the ripple unaware PLM to obtain the total flat noise $G_{\text {Noise_flat_p }}$. Then we train our model and obtain $\odot$ and use that to find the ripple noise penalty on the new connection $G_{\text {ripple_p }}(l)=\odot(f(l))$, and estimate the total noise with ripple $G_{\text {Noise_flat_p }}+\Theta(f(l))$. The estimation error will be identified once we establish the connection, monitor its values $Y_{p}(l)$ and compare it to that.

\section{Results and Discussion}

We considered the DT topology with 12 nodes and 40 bidirectional links whose lengths range from 48 to $458 \mathrm{~km}$ as shown in Fig 4a. We assumed 4 traffic loads of [100, 200, 300, 400] total connections with uniformly chosen sourcedestination having fixed symbol rate of $32 \mathrm{GBaud}$ and allocated 3 spectrum slots $(3 * 12.5 \mathrm{GHz}=37.5 \mathrm{GHz})$.
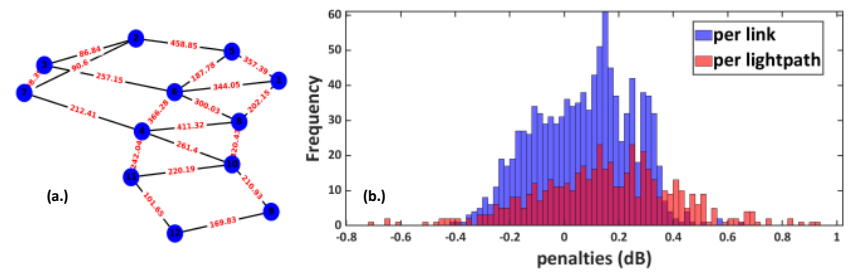

Fig 4. (a) DT 12 network topology, (b) penalty distribution.

We generated and assigned per span EDFA gain profile by randomly applying time shifting and amplitude scaling to experimentally obtained gain ripple dataset, $G_{i}(\lambda)$ (see Section 2 ). We assume that we have a set of connections established (according to the load) and monitored. Monitoring in our simulations is performed with a ripple aware PLM (extended GN) model, which give us $Y(P)$. We assume a stable network state, where we have a specific set of connections established and we want to establish a set of new connections. To do so we divide connections in two sets, $90 \% / 10 \%$, the training and testing datasets, respectively. The training set is assumed to be the established connections $P$ and the testing set the connections to be established. We use the ripple unaware PLM (GN) model to obtain $Q(P)$ depending on the attributes $P$ of the established connections. By subtracting $Q$ and $Y$, we obtain the penalty vector $E$ (ripple noise based penalty estimator block in Fig. $3 b$ ). The $E$ vector distributions are depicted in Fig. $4 \mathrm{~b}$ for 100 connections, which clearly shows the error values of $\sim 1 \mathrm{~dB}$. The penalties are distributed in positive and negative sides depending upon the ripple values. Positive/negative penalties result in upper/lower bound for design margins and we call them as, "high/low margin". We evaluated several ML assisted regression techniques to fit $\odot$ on $E$, such as linear fitting, quadratic, polynomial fitting, support vector machine (SVM) etc. In the presented results we used polynomial regressions of degree 4 that achieved maximum Mean Squared Error (MSE) of 4.5E-2 on predicted OSNR with load of 100 connections as shown in Fig. 5a. With increase in load from 100 to 400, max MSE converge to a value of $\sim 4 \mathrm{E}-3$. Results presented here are averaged over 200 iterations at each load.
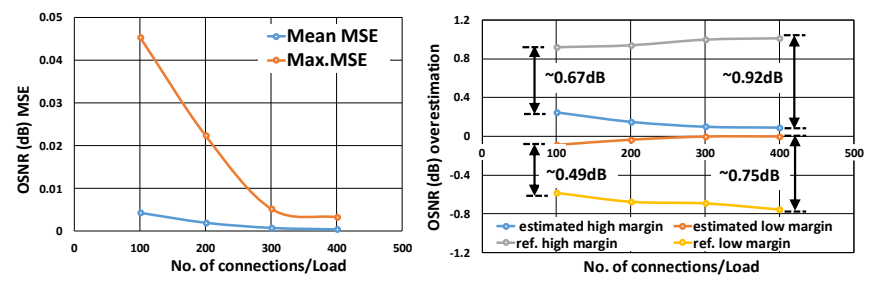

Fig 5. Effect of load variation on, (a) $\operatorname{OSNR}(\mathrm{dB}) \operatorname{MSE}(\mathrm{b})$ Max. overestimation error.

For the above set of simulations, the maximum used peak to peak ripple intensity among all span EDFAs is about $\pm 0.5 \mathrm{~dB}$, which results in a reference margin (design margin $_{1}$ ) of $1.02 \mathrm{~dB}$ at a load of 400 connections. Fig. 5b shows the maximum overestimation error on OSNR estimation, relative to Fig. 5a. This overestimation is the reduced estimated high and low margin (design $\operatorname{margin}_{2}$ ). For high margin, it is found to be $0.08 \mathrm{~dB}$, yielding a $\sim 0.92 \mathrm{~dB}$ margin reduction with 400 connections. For low margin, this reduction value is $\sim 0.75 \mathrm{~dB}$ as the distribution of penalties is less for low margin side.

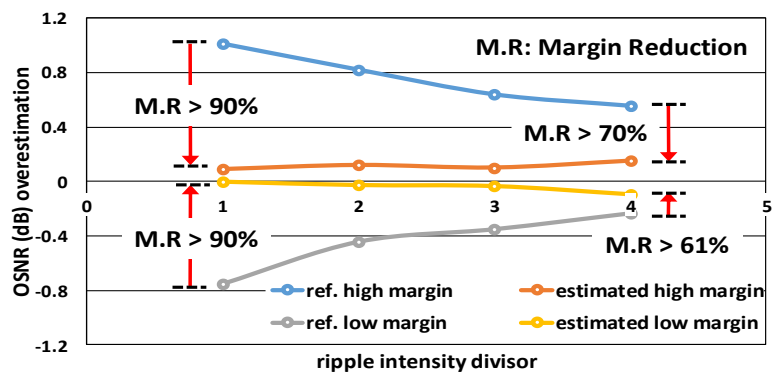

Fig 6. New margin for different intensities of peak to peak gain ripple among all EDFAs with reference as $\pm 0.5 \mathrm{~dB}$.

We also varied the gain ripple intensity (we divided the gain ripple profiles by a factor of 1 to 4 , resulting in peak-to-peak fluctuations of $\pm 0.5 \mathrm{~dB}$ to $\pm 0.125 \mathrm{~dB}$ ) and estimated the high and low margins at a fixed load of 400 connections. We observe in Fig. 6, a reduction of $>90 \%$ on both high and low margin for peak to peak intensity fluctuations of $\pm 0.5 \mathrm{~dB}$. For low values of peak to peak ripple of $\pm 0.125 \mathrm{~dB}$, high and low margin reduction varies from $60 \%$ to $70 \%$ respectively.

\section{Conclusion}

We proposed a ML method to estimate the noise penalty generated by EDFAs gain ripple. Leveraging that, we estimated the QoT accurately for new connection with $>90 \%$ reduction in the related margin.

\section{Acknowledgements}

The authors would like to thank Dr. Josep M. Fabrega and technical staff of CTTC ONS lab for providing lab facilities. This work is a part of ONFIRE project supported by EU Horizon 2020 research and innovation programme under the Marie Skłodowska- Curie grant agreement No. 765275. 


\section{References}

[1] V. Lopez and L. Velasco (Editors):' Elastic Optical Networks. Architectures, Technologies, and Control', Springer, 2016.

[2] E. Seve, et al.:' Learning Process for Reducing Uncertainties on Network Parameters and Design Margins' IEEE/OSA JOCN, 2018, 10, (2), pp. A298-A306

[3] L. Barletta, et al.:' QoT Estimation for Unestablished Lighpaths using Machine Learning' OFC, Los Angeles, CA, USA, 2017

[4] I. Sartzetakis, et al.:' Accurate quality of transmission estimation with machine learning' IEEE/OSA JOCN, 2019, 11, (3), pp. 140-150

[5] Y. Pointurier :' Design of Low-Margin Optical Networks ' IEEE/OSA JOCN, 2017, 9, (1), pp. A9-A17

[6] K. Ishii, et al.:' Wavelength Assignment Dependency of AGC EDFA Gain Offset under Dynamic Optical Circuit Switching Learning' OFC, San Francisco, CA, USA, 2014 [7] https://www.cisco.com/c/en/us/td/docs/optical/15000r7_0/ dwdm/planning/guide/70epg/d7ina.html

[8] L Qiao, et al.:' Erbium Doped Fiber Amplifier with Passive Temperature Compensation' OFC, Los Angeles, CA, USA, March 2017

[9] S. Zhu, et al.:' Machine Learning Based Prediction of Erbium-Doped Fiber WDM Line Amplifier Gain Spectra' ECOC, Rome, Italy, September 2018

[10] Y. You, et al.:' Machine Learning-Based EDFA Gain Model Spectra' ECOC, Rome, Italy, September 2018

[11] W. Mo, et al.:' Deep-Neural-Network-Based Wavelength Selection and Switching in ROADM Systems' IEEE/OSA JOCN, 2018, 10, (10), pp. D1-D11

[12] K. Ishii, et al.:' Experimental Investigation of Gain Offset Behavior of Feedforward-Controlled WDM AGC EDFA Under Various Dynamic Wavelength Allocations' IEEE Photonics Journal, 2016, 8, (1)

[13] M. Filer, et al.:' Multi-vendor Experimental Validation of an Open Source QoT Estimator for Optical Networks' IEEE JLT, 2018, 36, (15), pp. 3073-3082

[14] P. Poggiolini, et al.:' A detailed analytical derivation of the GN model of non-linear interference in coherent optical transmission systems' arXiv:1209.0394, 2012

[15] K. Christodoulopoulos, et al.:' ORCHESTRA-Optical performance monitoring enabling flexible networking," ICTON, Budapest, Hungary, July 2015 